$\begin{array}{ll}\text { le portiQue } & \text { Le Portique } \\ \text { Revue de philosophie et de sciences humaines }\end{array}$

16 | 2005

Sartre. Conscience et liberté

\title{
Ontologie, morale, histoire
}

\section{Alain Flajoliet}

\section{(2) OpenEdition}

\section{Journals}

\section{Édition électronique}

URL : http://journals.openedition.org/leportique/735

DOI : 10.4000/leportique.735

ISSN : $1777-5280$

\section{Éditeur}

Association "Les Amis du Portique"

Édition imprimée

Date de publication : 1 septembre 2005

ISSN : 1283-8594

\section{Référence électronique}

Alain Flajoliet, « Ontologie, morale, histoire », Le Portique [En ligne], 16 | 2005, mis en ligne le 15 juin 2008, consulté le 25 mars 2021. URL : http://journals.openedition.org/leportique/735 ; DOI : https:// doi.org/10.4000/leportique.735

Ce document a été généré automatiquement le 25 mars 2021.

Tous droits réservés 


\title{
Ontologie, morale, histoire
}

\author{
Alain Flajoliet
}

À Gérard Wormser

1 Nous voudrions présenter ici, dans le prolongement d'un travail récent ${ }^{1}$, une réflexion sur la manière dont chez Sartre s'articulent - difficilement - ontologie, morale et pensée de l'histoire, sur une période s'étendant des écrits de jeunesse aux Cahiers pour une morale de $1947-1948^{2}$. Les difficultés posées par cette articulation sont de deux ordres. En premier lieu - nous traiterons brièvement de ce point dans une première Partie - le concept même de morale sous horizon ontologique fait lever des difficultés considérables dans la pensée sartrienne, depuis les années d'études jusqu'à L'Être et le Néant. En second lieu, en 1947-1948, Sartre tente d'ouvrir un nouveau chemin vers le problème moral en modifiant profondément les rapports de cette dernière avec l'ontologie. Désormais l'agir humain, devenu "créateur ", soumet l'être au lieu de se soumettre à lui. Ce tournant fera l'objet de notre seconde Partie. Enfin nous analyserons, dans une troisième Partie, comment ce nouveau chemin se relie à l'émergence d'une théorie de l'histoire comme «totalité détotalisée ».

1. La subordination de l'agir à l'être. Les impasses du premier chemin1.1. L'impossible salut par l'art

2 La conviction d'un possible salut par l'art, pour l'homme en proie à la contingence, a une signification indissolublement morale et métaphysique. Métaphysique puisqu'elle signifie la possibilité d'annuler la contingence de l'existence humaine en la transmuant, par la création de l'œuvre éternellement belle, en essence idéalement nécessaire. Morale puisque cette transmutation, comme l'explique Sartre dans ses Carnets de la drôle de guerre, dessine un idéal de vie à la fois belle et bonne. Cependant la crise existentielle et philosophique du milieu des années trente, en détruisant la croyance en l'authentique possibilité de cette transmutation, désormais réduite à une pure fiction, oblige Sartre à « ranger comme il le dit dans Les Mots, l'impossible Salut au magasin des accessoires " ${ }^{3}$, Roquentin, à la fin de La Nausée, dans un "apologue » ironique, moque ses anciennes croyances d'une manière qui évoque clairement une sorte d'autocritique de l'auteur du roman. Toutefois les dernières lignes du livre installent l'ambiguïté, puisque Roquentin décide finalement d'écrire... un roman relatant une aventure «belle 
et dure comme de l'acier " ${ }^{4}$. Autant dire que l'illusion semble se réintroduire au cœur même de l'ultime prise de conscience critique.

Dans $L a$ Transcendance de l'ego, rédigé à Berlin simultanément à l'avancée du factum sur la contingence qui va devenir La Nausée, les perspectives morales sont plus nettement éloignées de la thématique du salut par l'art, tandis que s'efface la métaphysique soustendant cette dernière, au profit d'une philosophie transcendantale de la spontanéité de conscience absolument non substantielle. Toutefois, en déterminant la conscience transcendantale comme non substantialité, Sartre réintroduit bon gré mal gré des considérations ontologiques, en ce sens que cette conscience pure, se recréant constamment ex nihilo sans jamais être aucunement, apparaît comme néant radical. Et cette nouvelle ontologie de la conscience comme "néant » ouvre des perspectives morales inédites, esquissées de manière discrète à la fin de l'article: à chacun d'affronter l'angoisse de n'" être ", comme spontanéité transcendantale, absolument rien, et de ne pas fuir cette affection dans l'identification rassurante mais illusoire à l'être (rôle social, caractère stable, vie prédéterminée par son telos, etc.).

\subsection{Métaphysique de la réalité-humaine et morale de l'authenticité}

4 Les Carnets de la drôle de guerre sont une œuvre protéiforme aux visées très complexes... On peut admettre, pour simplifier, qu'ils constituent un tournant allant d'une phénoménologie transcendantale inspirée des Ideen... I de Husserl à une ontologie issue d'Être et temps de Heidegger. Simultanément, ils tentent d'élaborer une nouvelle morale, dont le sens n'est pas nettement fixé, précisément parce que les perspectives ontologiques elles-mêmes sont hésitantes. S'agit-il d'une « métaphysique de la réalitéhumaine", dans le fil de l'analytique existentiale d'Être et temps (moyennant les contresens bien connu à la fois sur le Dasein interprété à la suite de Corbin comme «réalité-humaine " et sur l'analytique existentiale pensée comme métaphysique) ? S'agit-il déjà de cette "ontologie phénoménologique » du maître ouvrage de 1943, dont les linéaments sont effectivement déjà présents dans nombre de fragments du Journal que tient Sartre? Ou même de cette philosophie transcendantale sous attracteurs ontologiques que constitue l'article sur l'Ego, et dont les traces sont encore perceptibles en 1939-1940 ? Malgré ces hésitations, on peut admettre qu'une préoccupation domine les Carnets : fonder sur une nouvelle métaphysique de la réalité-humaine une nouvelle morale dépassant les convictions ontologiques et morales d'avant guerre dont le contenu désormais est critiqué pour n'avoir fourni à Sartre que des règles de vie " inauthentiques ». Les catégories permettant de penser le sujet de la nouvelle morale la " réalité-humaine ", la "liberté » et sa «situation " - restent ontologiques (plus précisément métaphysiques) et cet état de chose compromet la nouvelle morale de l'« authenticité » et de l'« inauthenticité ».

5 Soldat sur le front de la drôle de guerre, Sartre vit douloureusement la désagrégation de sa manière de vivre d'avant-guerre (qu'il qualifie maintenant d'« inauthentique »), ainsi que la nécessité de reconstruire, au sein des bouleversements provoqués par le conflit mondial, une nouvelle existence «authentique». C'est Être et temps (mésinterprété comme "métaphysique de la réalité-humaine »), qui lui offre le cadre ontologique de sa réflexion sur l'authenticité et l'inauthenticité. Dans les Carnets, l'inauthenticité présente deux aspects.

6 Le premier, c'est la non assomption de la facticité. «L'inauthenticité, affirme Sartre en ce sens, consiste à se chercher un fondement pour "lever" l'irrationalité absurde de la facticité ${ }^{5}$. 
7 Mais en outre l'« inauthenticité », c'est aussi la non assomption de la liberté: «la réalité-humaine se voile [...] par lassitude le fait qu'elle est condamnée à se motiver elle-même ${ }^{6}$.

8 Le problème, c'est que la nouvelle métaphysique de la réalité-humaine compromet tout autant qu'elle rend possible la nouvelle morale de l'authenticité. Métaphysiquement (au sens que ce terme possède en 1939-1940), la réalité-humaine " est » un néant situé et fini, tout en étant habitée d'un incoercible désir de fuir ce néant qu'elle " est ». Or l'authenticité pour sa part est définie ainsi : « [...] réaliser pleinement son être-ensituation [...], avec cette conscience profonde que par la réalisation authentique de l'être-en-situation, on porte à l'existence plénière la situation d'une part et la réalitéhumaine d'autre part " ${ }^{7}$. Comment articuler dès lors l'élucidation métaphysique de la réalité-humaine comme fuite éperdue devant son statut de néant situé et fini, et l'exigence d'authenticité comme assomption résolue par cette même réalité-humaine de son statut de liberté irréductiblement située? La difficulté se noue autour du concept de « valeur».

9 L'élucidation de la valeur passe par celle de l'être de la réalité-humaine : «La réalitéhumaine, écrit Sartre, est d'un type existentiel tel que son existence la constitue sous forme de valeur à réaliser par sa liberté ${ }^{8}$. L'interprétation de ce "type existentiel » singulier qu'est l'homme s'appuie alors (de manière assez artificielle) sur l'analytique existentiale heideggérienne, où le Dasein est déterminé comme fondamental « à dessein de soi ». Dans les Carnets, la réalité-humaine est comprise comme projetant originairement la "valeur» suprême d' être substance ", ou "d'être son propre fondement ${ }^{9}$. Mais cette valeur qui dès lors hante tout le cours de vie de la réalitéhumaine, ainsi métaphysiquement comprise, ne rend-elle pas radicalement impossible toute attitude existentielle authentique, si l'authenticité consiste à s'assumer comme liberté située, c'est-à-dire comme néant facticiel et fini ? ${ }^{10}$ Inversement, si l'authenticité, comme attitude existentielle suprêmement valorisée d'assomption de soi comme liberté située, a un sens, comment peut-on affirmer qu'au cœur même de la réalitéhumaine prise dans ce qui la constitue le plus fondamentalement, se trouve nécessairement une fuite devant son statut ontologique de néant facticiel et fini ?

Le projet d'être soi dans la forme de l'en soi peut bien être par principe irréalisable, il demeure une possibilité constitutive de la réalité-humaine et c'est qui rend problématique toute la morale de l'authenticité des Carnets. Comment en effet exiger de l'être humain des conduites où, s'assumant comme liberté située, il s'oppose pour ainsi dire à ce qui le constitue ontologiquement? Ce problème est au cœur de L'Être et le Néant.

1.3. Les « perspectives morales » dans l'ontologie phénoménologique1.3.1. Introduction

11 L'«ontologie phénoménologique» de 1943 s'efforce en principe de conserver, conformément aux injonctions d'Être et temps, une neutralité morale. Les « perspectives morales » se situent donc au-delà de l'ontologie (tout comme les perspectives " métaphysiques ») et ne sont abordées qu'à la toute fin de l'ouvrage ${ }^{11}$. Une des raisons essentielles de la différence de principe entre morale et ontologie est que la première se place au niveau des conduites existentielles concrètes, tandis que la seconde élucide les structures existentiales constitutives du pour soi comme tel. Toutefois l'ontophénoménologie donne des indications contraignantes à la future morale, car toutes les déterminations ontologiques du pour soi ne sont pas annulées lorsqu'on veut fonder la morale. Cette dernière doit assumer en particulier le fait que tout pour soi est un ipse 
hanté par la valeur de toutes la valeurs : lui-même dans la forme de l'en soi. Que signifie dès lors vivre concrètement « bien » ou " mal » cette structure ontologique constitutive de tout pour soi? Il n'est pas aisé de déterminer plus avant cette distinction entre « bonnes » et « mauvaises » conduites.

L'opposition entre «bonne foi » et "mauvaise foi » ne nous est pas ici d'un grand secours, elle est établie dans le cadre de l'ontologie phénoménologique - elle signifie la distinction entre deux types de conduites existentielles concrètes à partir desquelles Sartre dégage la première structure existentiale de la "présence (à) soi ». Elle ne possède donc pas en principe de signification morale: "il est indifférent d'être de bonne ou de mauvaise foi ${ }^{12}$. Quant à l'opposition entre authenticité et inauthenticité, Sartre n'en fait plus usage en $1943^{13}$. Toutefois, si l'on se reporte aux trois dernières pages du maître ouvrage consacrées à esquisser les "perspectives morales", deux indications positives apparaissent. D'une part l'affirmation que l'attitude morale doit consister dans une rupture avec l'esprit de sérieux. D'autre part la thèse selon laquelle l'angoisse " découvre à l'agent moral qu'il est l'être par qui les valeurs existent " ${ }^{14}$. Le lien entre ces deux affirmations n'est pas, à vue de pays, très évident.

La critique de l'esprit de sérieux renvoie à l'élucidation des fondements ontologiques de la "psychanalyse existentielle ", où Sartre, classant les principaux types existentiels d'humanité, fait apparaître, à côté du savant et de l'artiste, le joueur. Le joueur : celui qui a décidé de faire de sa vie une scène de théatre où aucune action n'est spontanée parce qu'on y est en perpétuelle représentation. L'angoisse renvoie de son côté à la thématique du choix originel que chacun nécessairement fait de lui-même, sans aucun acquis ni point d'appui, c'est-à-dire dans une absolue liberté.

Sartre manifeste ainsi le lien entre l'esprit de jeu et la conscience angoissée de la liberté inconditionnée : «Le jeu, [...] comme l'ironie kierkegaardienne, délivre la subjectivité. Qu'est-ce qu'un jeu, en effet, sinon une activité dont l'homme est l'origine première, dont l'homme pose lui-même les principes et qui ne peut avoir de conséquences que selon les principes posés? Dès qu'un homme se saisit comme libre et veut user de sa liberté, quelle que puisse être par ailleurs son angoisse, son activité est de jeu ${ }^{15}$ » et le monde n'a pour lui que peu de réalité.

Ces remarques contiennent deux difficultés. D'abord il n'est pas certain qu'elles s'accordent bien avec ce que Sartre affirme par ailleurs dans les premières lignes de son étude des "perspectives morales " où s'achève L'Être et le Néant: "l'éthique ", dit-il alors, implique une réalité-humaine en situation et pleinement responsable ${ }^{16}$. Comment concilier l'esprit de responsabilité avec l'esprit de jeu? La valorisation de l'esprit de jeu ou, si l'on préfère, l'affirmation de la suprême valeur des conduites exprimant une liberté inconditionnelle, ne détruisent-elles pas à sa racine la notion même de responsabilité, clé de la morale sartrienne de 1943 ? Cette difficulté n'est peut-être pas insurmontable, dès lors qu'on redéfinit totalement (comme le propose Sartre) le concept de responsabilité pour le rendre compatible avec celui d'une liberté humaine absolue. On peut toutefois se demander ce qu'il reste du concept de responsabilité lorsqu'on qualifie cette dernière d'absolue ${ }^{17}$. Il y a en outre une seconde difficulté. La morale de l'esprit de jeu doit envelopper, pour ne pas valoriser un irréalisable de principe, la croyance en la possibilité réelle (et non pas vide), pour tout individu, de s'évader de sa situation par la vertu de son agile conscience absolument libre. Or cette possibilité semble bien contredire l'affirmation par l'ontologie phénoménologique d'une finitude et d'une facticité irréductibles de l'ipse engagé dans le 
monde. Examinons ces deux difficultés, en analysant successivement la théorie de la responsabilité et celle des types de vie préférables.

1.3.2. Le choix originel et la responsabilité absolue de la réalité-humaine

Avec la Quatrième Partie de L'Être et le Néant et l'effort pour élucider l'action humaine libre et située, Sartre se rapproche à l'évidence de questions proprement morales. Mais il ne s'autorise d'affronter explicitement ces dernières que dans un bref passage consacré à la responsabilité ${ }^{18}$. Pour le reste il s'en tient à une analyse ontico-ontologique.

17 L'idée que la réalité-humaine se choisit radicalement en renouvelant son choix à chaque instant dans une sorte de réinvention constante et radicale de soi, signifie d'abord qu'aucune morale de l'impératif catégorique ou du devoir absolu n'a de sens, puisqu'une telle morale contredit directement l'absoluité d'une telle recréation en soumettant la liberté à la loi. La liberté humaine, écrit Sartre, « est totale et infinie " ${ }^{19}$. Nous voici bien en présence de la première difficulté signalée. La formule sartrienne est en effet pour le moins déroutante, en raison du complet effacement qu'elle opère de la finitude et de la facticité de l'ipse, que pourtant les deux premières Parties de L'Être et le Néant ont réaffirmé constamment avec la plus grande netteté. Dans la morale issue de la métaphysique de la liberté absolue, la valeur suprême ne peut consister, pour la réalité-humaine, qu'à recréer à chaque instant son existence entièrement à partir d'elle-même, tandis que, simultanément, toutes les conduites ou elle se fait annoncer ce qu'elle a à faire par l'Autre (Dieu, tel ou tel rôle social, familial, professionnel, etc.) apparaissent comme anti-valeurs.

Dès lors, la valeur morale suprême d'absolue responsabilité ne paraît constituer qu'une exigence vide et ineffectuable : comment éviter que l'action réelle ne s'insère dans le jeu des causes mondaines relevant de l'extériorité d'inertie, de sorte que notre responsabilité soit à chaque instant mesurée par la situation où notre liberté « s'engrène » ? 20

19 L'exigence métaphysique de perpétuelle recréation de soi inscrite au cœur de la réalitéhumaine, dans L'Être et le Néant, est la clé des paradoxes accumulés par la doctrine morale de la responsabilité absolue. Citons celui-ci: «en un certain sens, je choisis d'être né ${ }^{21}$. Ou celui là : chaque citoyen est entièrement responsable de la guerre dans laquelle son pays s'est engagé ${ }^{22}$. Et encore : tout « ce qui m'arrive, m'arrive par moi » ${ }^{23}$. Certes la réalité-humaine agit nécessairement dans une «situation » déterminée - nier cela détruirait purement la doctrine de la finitude et de la facticité du pour soi. Mais cette situation ne contribue nullement à modeler le choix originel de soi-même, tout au plus est-elle pour ce dernier l'occasion de sa libre auto-constitution. Toutefois c'est aussi par un second biais que l'ontologie fournit à la future morale des indications qui posent à cette dernière de redoutables problèmes.

1.3.3. La psychanalyse existentielle et les perspectives morales

Dans la Quatrième Partie de L'Être et le Néant, Sartre ne se contente pas d'exposer une théorie de l'entière responsabilité humaine sur la base de l'ontologie (sous attracteur métaphysique) du choix originel, il pose les fondements ontologiques de la "psychanalyse existentielle", qui à leur tour vont développer d'importantes implications morales. «La psychanalyse existentielle, affirme-t-il dans une formule quelque peu inexacte, est une description morale » ${ }^{24}$. Examinons cette affirmation.

21 La psychanalyse existentielle se fonde sur le concept ontologique très concrétisé et très dérivé de réalité-humaine désirante. La classification des principales espèces du désir humain (désir d'avoir, de faire et d'être) permet d'esquisser une typologie des modes 
d'existence fondamentaux (le savant, l'artiste, le joueur), typologie qui à son tour rend possible une morale dans la mesure où à chaque type d'existence est conféré une certaine valeur.

Concernant le désir de faire, Sartre affirme qu'il se réduit immédiatement au désir d'avoir - ou plus exactement d' " appropriation » : on fait quelque chose pour posséder quelque chose. Mais à son tour le désir d'avoir se réduit au désir d'être, car il s'interprète comme hanté par une impossible "création" de l'objet désiré où se parachèverait l'appropriation dans sa forme excellente et parfaite. L'enchainement des deux réductions est capital : le désir de faire, in fine, se subordonne au désir d'être dans la forme excellente de l'être divin se récréant dans l'être. Ainsi replacée dans l'orbe du désir d'être, l'action humaine ne peut avoir aucune autonomie, et la morale, condamnée une fois de plus à se déployer dans l'ombre portée de l'ontologie, se retrouve prise dans d'inextricables difficultés.

Examinons les trois manières fondamentales possibles de mettre en œuvre existentiellement ce désir existential-ontologique d'être Dieu créateur auquel se ramènent tous les désirs humains concrets quant leur visée ultime (essentiellement possessive, donc). Laissons de côté le savant et l'artiste, et concentrons notre analyse sur le type existentiel auquel Sartre semble bien donner la plus grande valeur, si l'on se reporte aux « perspectives morales » de la fin du livre : le joueur.

On comprend en effet assez rapidement que ce dernier type existentiel, par rapport aux deux premiers, est singularisé par la valeur tout à fait spécifique que lui confère la description sartrienne. En effet, la remarque selon laquelle, le jeu, et lui seul, s'oppose à "l'esprit de sérieux " ${ }^{25}$, fait immédiatement dresser l'oreille au lecteur tant soit peu averti. Ce lecteur se souvient aisément que, dans les premières pages de La Force de l'âge, consacrées aux années 1929-1931, S. de Beauvoir explique combien elle et Sartre haïssaient "l'esprit de sérieux", inventant constamment, pour mettre la réalité à distance, des comédies, parodies, apologues, jeux de mots, fables, son compagnon se révélant particulièrement doué dans ce genre d'activités ${ }^{26}$. Tout ce que nous savons des années d'études de Sartre confirme la haine de ce dernier pour l'esprit de sérieux : goût du canular, de la provocation antimilitariste, raillerie systématique des autorités enseignantes et administratives de l'E.N.S. Mais que faut-il entendre plus précisément par « esprit de sérieux »?

La définition qu'en donne Sartre au début L'Être et le Néant nous ramène directement à la morale. L'esprit de sérieux, écrit-il, « saisit les valeurs à partir du monde et [...] réside dans la substantification rassurante et chosiste des valeurs ${ }^{27}$. On en déduit aisément que l'esprit de jeu est étroitement lié à l'appréhension angoissée de notre absolue liberté par qui les valeurs existent. La morale devient une sorte de « conversion ${ }^{28} \mathrm{de}$ l'esprit de sérieux à l'esprit de jeu.

Dans l'esprit de sérieux, l'action est effectuée comme si elle était suscitée en quelque sorte par une valeur purement objective. C'est de là que vient la croyance (illusoire) de l'individu en l'importance de sa tâche. Dans l'esprit de jeu en revanche, l'individu agit avec une sorte de détachement ironique, sans jamais se laisser prendre à la soi-disant importance de ce qu'il fait, car il a conscience que ce qu'il choisit de faire, il pourrait tout aussi bien choisir de ne pas le faire. La conscience morale enveloppe eo ipso une indifférence aux buts concrets de l'action. (La morale de l'esprit de jeu rejoint en ce sens certaines implications de la métaphysique de la liberté absolue). 

instant de s'engager sérieusement dans aucune des actions qu'il entreprend. Mais comme nous venons de le rappeler, l'articulation du choix existentiel ce type de vie avec ce que l'ontologie phénoménologique affirme du désir appropriatif d'être Dieu, fait lever d'inextricables difficultés. Car de deux choses l'une. Soit, comme le dit Sartre, la liberté, dans son effort proprement moral pour se retourner sur la valeur, échoue parce que, " quoiqu'elle fasse », elle "emporte avec elle cette valeur» qui la ressaisit toujours "par derrière ${ }^{29}$. Fin de l'exigence morale. Soit la liberté réussit à se vouloir liberté, mais alors elle met un terme au règne de la valeur. Fin de l'ontologie de la réalité humaine.

2. Ouverture d'un second chemin. Le concept de création et la subordination de l'être à l'agir

31 Les Cahiers pour une morale proposent, en 1947-1948, de construire une nouvelle morale selon un plan esquissé de manière très ferme ${ }^{30}$. Elle se serait divisée en deux «Sections ». La première portant pour titre : «Plan d'une morale ontologique ». La seconde Section, sans titre, aurait traité manifestement de l'histoire humaine. Entrons dans le contenu de ces deux Sections.

La première, explique le plan, aurait analysé l'« aliénation » de la liberté humaine ${ }^{31}$, en dégageant en particulier ses trois principales espèces (aliénation par la nature humaine, par le devoir et le droit, et par les valeurs réifiées), mais aussi la forme même de l'Aliénation : être soi-même comme un Autre (Ego ou Dieu). Elle aurait décrit le «monde de l'aliénation » (monde des prières des exigences, de la violence ${ }^{32}$...). Elle aurait fourni une théorie des «valeurs» en les classant selon le degré de liberté créatrice qu'elles autorisent ${ }^{33}$. Enfin et surtout, elle aurait expliqué comment une « conversion » était possible, du monde de l'aliénation au monde de la liberté. Tout ceci se relie assez clairement au projet d'une morale "ontologique», en ce sens que la " conversion" semble bien s'interpréter comme réappropriation par l'individu d'une capacité créatrice perdue dans l'aliénation. Il est donc tout à fait logique que Sartre ait songé à intituler cette première Section: "morale ontologique ». Morale de la « conversion », comme tous les projets de morale examinés jusqu'ici, réinvestissant le 
concept de réflexion «pure » ou «non complice " ${ }^{34}$, mais en accentuant désormais les implications morales de ce concept, ce qui n'était nullement le cas dans L'Être et le Néant ${ }^{35}$. Car dans L'Être et le Néant la réflexion pure signifie seulement l'«intuition fulgurante et sans relief " ${ }^{36}$ qui donne l'être de l'irréfléchi sans l'altérer, c'est-à-dire sans l'objectiver. (Par opposition, la réflexion «impure» objective cet être en Ego psychique ${ }^{37}$ ). Dans le texte de 1947-1948 maintenant publié, c'est le "Second Cahier » qui correspond le mieux à ce projet de déployer une morale de la "conversion » à la vie créatrice. $\mathrm{Ce}$ « Second Cahier » présente trois parties principales :

D'abord une partie formée de fragments désordonnés ${ }^{38}$, réévoquant d'une part la question de l'histoire qui était au centre du «Premier Cahier»; et d'autre part analysant des thèmes qui se relient à la morale ontologique (en particulier le thème de la création).

Puis vient le plan de la morale à construire ${ }^{39}$.

Enfin une troisième partie étudie la «conversion» à partir de ce concept de « création » qui constitue désormais le fondement de la future morale ontologique ${ }^{40}$.

Pour ce qui regarde la seconde Section du plan, elle prend pour thème, comme nous le disions, l'histoire humaine. Ce qui conduit Sartre d'une part, à batailler contre la conception « dialectique » de l'histoire ${ }^{41}$. D'autre part à esquisser une lecture originale de l'histoire humaine comme "oppression» et possibilité d'une «libération» de l'homme dans cet ultime moment qu'il appelle "l'Apocalypse " ${ }^{42}$. De même que l'ontologie phénoménologique de 1943 ouvrait des "perspectives" sur une morale ontologique, de même maintenant la rénovation du projet de morale ontologique ouvre des «perspectives historiques ». Toute la difficulté consiste à saisir l'articulation entre la Section I et la Section II du plan proposé par Sartre dans ses Cahiers, autrement dit à comprendre comment la morale ontologique ouvre des perspectives historiques. Car cette articulation ne va nullement de soi, pas plus qu'en 1943 l'articulation de l'ontologie phénoménologique et des perspectives morales n'allait de soi. apparente de la situer dans le prolongement de ses tentatives antérieures dans lesquelles, nous l'avons vu, la pensée de l'agir était toujours étroitement liée à une doctrine de l'être. De fait, d'assez nombreuses analyses ontologiques anciennes surtout issues de L'Être et le Néant - sont apparemment réinvesties dans les Cahiers. Par exemple, l'interprétation de la conversion morale à partir des concepts de réflexion " complice» ou «impure » et de réflexion "non complice» ou "pure». Mais aussi l'analyse d'autrui comme regard pétrifiant ${ }^{43}$. Ou celle du manque constitutif du pour soi ${ }^{44}$.

Toutefois, à y regarder de plus près, un tournant est bien pris en 1947-1948 par rapport à ce qu'on peut appeler un "projet de morale ontologique », parce que désormais les concepts de «création » et d' " aliénation » deviennent la clé de voûte de ce dernier (ce qui n'était nullement le cas dans les projets antérieurs). La «conversion» morale est maintenant comprise comme désaliénation de l'être humain, c'est-à-dire arrachement à l'extériorité d'inertie et reconquête d'une liberté créatrice ${ }^{45}$. La "Section I » du plan des Cahiers esquisse ce qu'aurait été l'élucidation de ce mouvement de conversion, et le Cahier deux développe de manière assez précise ce qui dans l'esquisse touche à l'idée de « conversion ». Il nous semble que le concept de « création » constitue l'une des clés du tournant de la pensée sartrienne après la seconde guerre mondiale, tournant que nous avons qualifié par ailleurs d'humaniste dans un article récent ${ }^{46}$ - aussi bien les deux 
aspects n'en font qu'un: c'est l'homme qui tend désormais à être défini comme créateur, alors que dans L'Être et le Néant la réalité-humaine peut bien être hantée en toutes ses actions par le Dieu créateur, elle échoue nécessairement à créer réellement quoi que ce soit dans le monde ${ }^{47}$. "Toute action est création", affirme péremptoirement Sartre à la première ligne de sa phénoménologie de l'action dans le Premier Cahier ${ }^{48}$. En devenant créateur l'agir humain tend à soumettre l'être à luimême. Sartre, en rompant avec la perspective - qui dominait jusqu'alors sa pensée, en particulier dans L'Être et le Néant ${ }^{49}$ - selon laquelle à l'inverse l'agir se subordonne à l'être, peut escompter deux bénéfices : échapper aux difficultés qui grevaient toutes ses tentatives d'élaborer une morale à partir de l'ontologie, et recentrer sa philosophie sur une anthropologie en s'éloignant des rivages de l'ontologie phénoménologique. La Conférence L'existentialisme est un humanisme de 1945, en dépit de ses faiblesses conceptuelles évidentes, laisse bien apparaître ce tournant «à deux faces»: a) recentrage de la philosophie sur la subjectivité humaine b) comprise comme « invention » perpétuelle de soi ${ }^{50}$.

Reste à savoir si ce concept d'humanité « créatrice » permet d'asseoir correctement la nouvelle morale ontologique et s'il ne fait pas surgir de nouvelles difficultés. D'abord dans son élucidation propre, qui est loin d'être claire dans les Cahiers. C'est le premier point que nous examinerons. Ensuite - second problème à analyser - dans ses rapports au concept d'aliénation. L'aliénation, sous l'effet de la doctrine de la liberté créatrice, tend à envahir la description de toutes les formes d'« exigence ».

\subsection{Conversion et création}

Quand on se reporte au plan fourni par Sartre, on voit d'abord que la morale ontologique réinvestit des concepts de l'ontologie phénoménologique. L'existant, affirme Sartre en ouverture, "manque d'être ${ }^{51}$, de sorte qu'il se projette vainement comme soi dans la forme de l'en soi. La "réification » comme " phénomène ontologique premier » est donc, affirme Sartre, la clé de «l'inauthenticité ». Rappelons que L'Être et le Néant ne faisait quasiment pas usage de l'opposition authenticité-inauthenticité, ni dans ses analyses proprement ontologiques - ce qui est tout à fait normal - mais même dans les quelques pages finales consacrées aux "perspectives morales ». L'opposition en revanche était constitutive du projet des Carnets de la drôle de guerre.

41 Autre innovation, l'introduction presque immédiate du concept d'« aliénation », définie comme nous l'avons vu comme "aspect social de la réification». Nous aurons à analyser plus avant l'idée d'aliénation, contentons-nous ici des quelques remarques nécessaires à l'analyse de l'idée de création. "Le monde de l'aliénation, écrit Sartre, c'est celui où on pense le Soi-même à partir de l'Autre ${ }^{52}$. Le concept d'aliénation n'était certes pas totalement absent de L'Être et le Néant ${ }^{53}$, mais son usage y était limité à l'élucidation de l'une des structures existentiales relativement dérivées du pour soi : le pour autrui. Ici, il devient le mode d'être originel de la réalité-humaine, son inauthenticité première.

Sartre précise que ce concept d'aliénation n'est pas le concept marxiste, car: "l'aliénation précède l'oppression " ${ }^{54}$. Précision importante parce qu'elle suggère que dans les Cahiers l'«aliénation" renvoie plutôt au projet de morale ontologique, et l'« oppression » au projet d'interprétation de l'histoire.

On peut d'autre part conjecturer qu'en faisant de l'aliénation le "porche» de sa nouvelle morale, Sartre tient à placer d'entrée de jeu cette dernière sous le signe de l'intersubjectivité, puisque l'aliénation implique la présence d'autrui qui pour ainsi dire 
me vole ma liberté (sans la détruire). Mais il n'est pas sûr que cela soit parfaitement cohérent avec la thématique, disons "individualiste", de la conversion à la vie créatrice ${ }^{55}$.

Toutes les fins dans la "morale ontologique" se déploient donc entre les deux extrêmes de l'aliénation totale - l'anti-valeur pour ainsi dire, la liberté entièrement ressaisie par l'être - et de la conversion à la liberté absolue se reprenant totalement sur son aliénation - la valeur suprême. Comme nous le disions, le concept dont Sartre se sert pour expliciter cette valeur suprême est celui de liberté créatrice et c'est ce point que nous devons maintenant analyser de plus près.

On doit d'abord constater que le concept de « création » est omniprésent dans la morale proprement ontologique de 1947-1948, alors que dans L'Être et le Néant il est rare ${ }^{56}$. Nous l'avons vu apparaittre essentiellement dans la psychanalyse existentielle : parmi les types existentiels analysés brièvement se trouve "l'artiste » défini par son désir de créer, désir irréalisable en raison du caractère contradictoire de l'idée de création (dont le modèle est en Dieu). Dans une ontologie phénoménologique pure, ce concept de création n'a pas de fonction autre que celle de concrétiser l'irréalisable « valeur » du soi dans la forme de l'en soi. Il peut par ailleurs jouer un rôle d'attracteur métaphysique de cette ontologie lorsque cette dernière se transforme en théorie de la réalité-humaine ${ }^{57}$ - c'est ce concept qui à l'évidence entraîne la phénoménologie ontologique de la liberté, comme nous l'avons vu, vers l'affirmation qu'existe dans toute réalité-humaine un choix inconditionné d'elle-même qu'elle réeffectue à chaque instant. Le concept de création appartenant totalement à l'histoire de l'onto-théologie, il n'est pas étonnant de le trouver dans des textes philosophiques ou psychologiques sartriens d'avant le tournant phénoménologique de Berlin (1933-1934). Il constitue par exemple la clé de voûte du Diplôme d'Études Supérieures de Sartre qui déploie une métaphysique de "l'esprit créateur» comme fondement de la faculté psychologique d'imaginer en l'homme. Comment se présente la création dans la nouvelle morale ontologique de 1947-1948?

La thématique de la création envahit le texte sartrien à partir de ce passage du "Second Cahier » intitulé : «La conversion » ${ }^{58}$. Partout, Sartre réaffirme que l'homme est "pouvoir créateur " ${ }^{59}$, que ce pouvoir s'efface dans l'aliénation et qu'on le reconquiert dans la conversion. Que signifie cette exigence de conversion, suprême valeur de la nouvelle morale ontologique? Essentiellement une rupture avec toute volonté d'être, qualifiée maintenant d'inauthentique. "Originellement, écrit Sartre, l'authenticité consiste à refuser la quête de l'être ${ }^{60}$. Cette affirmation constitue une prise de distance (plus ou moins explicite) avec L'Être et le Néant, puisque désormais, en l'homme, les actions ne sont plus assignées à l'être (on agit pour être, est-il dit dans le maître ouvrage de $1943^{61}$ ). Elles peuvent s'autonomiser radicalement - précisément dans l'authenticité - par rapport à toute volonté d'être, en devenant actions créatrices s'inventant totalement elles-mêmes hors de toute assignation ontologique préalable. Dans l'attitude authentique, précise Sartre, non seulement la réflexion, mais même les sentiments et croyances deviennent des "entreprises " ${ }^{62}$ - on se fait amoureux comme on se fait croyant. Laissons pour le moment l'esquisse d'analyse de cette notion d'« entreprise » que propose ici Sartre, parce qu'elle s'écarte significativement de la notion de création ${ }^{63}$. Intéressons-nous à la reprise du thème ancien de la conscience révélatrice de l'être à partir du concept de « dévoilement » authentique. 

l'être (au premier chef de celui qu'elle n'est pas), constitue une novation majeure. "Dévoiler », dit Sartre, c'est «créer ce qui est » ${ }^{64}$. Cette formule nous rapproche fort de l'intuition intellectuelle kantienne, comme Sartre le suggère lui-même dans « Qu'est-ce que la littérature ?» ${ }^{65}$. Sartre assume explicitement l'écart de sa nouvelle doctrine de la création dévoilante par rapport aux analyses de L'Être et le Néant - l'inédit Vérité et existence confirme les orientations $\mathrm{du}$ "Second Cahier » que nous suivons ici ${ }^{66}$. Mais cette réinterprétation créatrice de la révélation ontologique, qui nous éloigne de la doctrine de la révélation originelle de l'être à la conscience par décompression en présence (à) soi ${ }^{67}$, nous plonge en plein paradoxe. Car Sartre est conduit à penser l'Être (ou l'en soi) comme « appel » lancé en direction du pour soi, qui y répond précisément par son dévoilement créateur. Reconnaissons qu'il paraît difficilement justifiable, d'une part de conférer une capacité d'«appel » à un en soi qui est ce qu'il est dans son indifférenciation, sa contingence et son ipséité radicale (Vérité et existence évoque en ce sens « la nuit de l'Être » $\left.{ }^{68}\right)$. D'autre part de conférer au pour soi la fonction quasi-divine de créer l'Être en le dévoilant ${ }^{69}$.

Et pourtant Sartre n'hésite pas à écrire : "C'est l'appel de l'Être (que le Pour-soi soit conscience de plus en plus d'Être). En même temps le Pour-soi prend conscience de soi comme destiné à faire qu'il $y$ ait de plus en plus d'Être, c'est-à-dire à manifester l'Être ${ }^{70}$. C'est donc la mission du Pour-soi que de "sauver» l'Être du Néant en le dévoilant : « Le Pour-soi surgit pour que l'Être devienne Vérité. Par là le Pour-soi a une tâche de quasi-création puisqu'il extirpe des ténèbres de l'indifférenciation ce qui par essence y retombe toujours ${ }^{71}$. On voit ici comment la situation se retourne par rapport aux séries d'apories relevées dans la première Partie de cet article : ce n'est plus l'ontologie qui rend problématique la construction d'une morale, c'est la morale qui pousse l'ontologie à formuler des thèses peu soutenables. "La morale, écrit Sartre par exemple, en libérant le Pour-soi de l'aliénation (qui fait de lui un inessentiel), rend à la conscience son existence d'absolu $"{ }^{72}$. Cela peut s'entendre, mais à condition de ne pas réintroduire trop vite dans cette désaliénation la notion d'un absolu créateur. Car l'absolu de conscience est en principe un absolu-relatif, ou, si l'on préfère, la liberté humaine est en principe finie et facticielle (située). Sur cette ligne de pensée, les Cahiers réinvestissent le concept d'« historicité » qui dans les Carnets de la drôle de guerre traduisait, en suivant H. Corbin, la "Geschichtlichkeit » de Sein und Zeit ${ }^{73}$. «Ainsi, écrit Sartre, je m'historialise en me revendiquant comme conscience libre d'une époque, ayant son avenir dans l'avenir de l'époque et ne pouvant manifester que cette époque, ne pouvant dépasser l'époque que si je l'assume en sachant que ce dépassement même de l'époque est d'époque et contribue à la faire " ${ }^{74}$. Moyennant la précision nouvelle que l'assomption par l'homme de lui-même comme liberté située est source de "joie», et moyennant aussi une très considérable déperdition de clarté dans l'élucidation des concepts de contingence, de gratuité, de finitude ${ }^{75}$, tout cela reste au plus près de la problématique ontologique de 1939-1940. Le problème est que simultanément Sartre maintient que l'absoluité de la liberté signifie son caractère créateur qui en fait une liberté quasidivine ${ }^{76}$.

Au total, la position des Cahiers apparaît flottante, prise entre les thèses de l'ancienne ontologie sur la facticité et la finitude irréductibles de la liberté humaine, et la nouvelle affirmation, issue du tournant anthropologico-moral, d'une liberté créatrice en l'homme. Évoquant le dévoilement de l'Être par le Pour-soi, Sartre écrit cette phrase 
qui n'est que l'expression même de la contradiction : «Ainsi retrouvons-nous mais dans l'humilité de la finitude, l'extase de la création divine $»{ }^{77}$.

Le concept de l'homme créateur entraîne, outre ces confusions et incertitudes dans l'analyse même de son sens, des difficultés dans la compréhension de l'intersubjectivité ${ }^{78}$. De même que l'interprétation de la révélation de l'en soi par la conscience comme dévoilement "créateur» laissait le lecteur perplexe, de même l'appréhension du rapport à autrui dans l'horizon du concept de création n'apparaît nullement clair. «Dans l'authenticité, écrit Sartre, je choisis de dévoiler l'Autre. Je vais aussi créer les hommes dans le monde ${ }^{79}$. Comment faut-il l'entendre ? Négativement, cela signifie en toute rigueur que l'on doit abandonner l'interprétation du pour autrui de L'Être et le Néant comme "Enfer » ${ }^{80}$ des regards pétrifiants. Mais Sartre ne s'y résout pas franchement, préférant affirmer, de façon manifestement inexacte: «Dans cet enfer déjà il y avait générosité et création ${ }^{81}$.

Positivement, le sens de cette réinterprétation de l'intersubjectivité comme rapport de libertés créatrices est à chercher, suggère Sartre ${ }^{82}$, du côté de la création artistique. C'est une allusion transparente à "Qu'est-ce que la littérature?", dont les Cahiers résument l'une des principales thèses. «Il y a différentes manières, dit Sartre, de saisir le but [de la liberté d'autrui] : si je le transcende simplement vers le mien propre, ce but devient chose. Il est absurde et contingent. Mais la contemplation de l'œuvre d'art nous permet de saisir comment je puis appréhender le but de l'Autre: l'œuvre d'art se présente à moi comme fin absolue, exigence et appel. Elle s'adresse à ma pure liberté et par là me révèle la pure liberté de l'autre " ${ }^{83}$. "Qu'est-ce que la littérature? » met plus fortement en relief, sur l'exemple de l'écriture littéraire, le lien entre cette reconnaissance mutuelle des libertés et le caractère créateur de ces dernières. (Ce qui, soit dit en passant, fait lever le soupçon que les Cahiers pourraient ne développer finalement qu'une morale d'écrivain). Dans ce texte, Sartre explique que tout écrivain désire, en créant, " dévoiler et produire à la fois " ${ }^{84}$ l'œuvre, entreprise contradictoire qui s'effondre sur elle-même tant que le lecteur n'entre pas en scène. «Il n'y d'art que par et pour autrui " ${ }^{85}$. C'est en effet par le lecteur que l'être de l'œuvre, vainement recherché par l'écrivain solitaire, advient par le lecteur. Et comme par ailleurs ce dernier contribue à la création de l'écrivain en faisant vivre le monde imaginaire à travers les mots et les phrases déposés sur le papier, Sartre peut écrire : « La lecture [...] semble la synthèse de la perception et de la création; elle pose à la fois l'essentialité du sujet et celle de l'objet; l'objet est essentiel parce qu'il est rigoureusement transcendant, qu'il impose de ses structures propres et qu'on doit l'attendre et l'observer; mais le sujet est essentiel aussi parce qu'il est requis non seulement pour dévoiler l'objet (c'est-à-dire faire qu'il $y$ ait un objet), mais encore pour que cet objet soit absolument (c'est-à-dire pour le produire). En un mot, le lecteur a conscience de dévoiler et de créer à la fois, de dévoiler en créant, de créer par dévoilement " ${ }^{86}$.

Cette analyse éclaire certains concepts fondamentaux de la morale de 1947-1978. Au premier chef celui d'« appel ». " Puisque la création, écrit Sartre, ne peut trouver son achèvement que dans la lecture, puisque l'artiste doit confier à un autre le soin d'accomplir ce qu'il a commencé, puisque c'est à travers la conscience du lecteur seulement qu'il peut se saisir comme essentiel à son œuvre, tout ouvrage littéraire est un appel. Écrire, c'est faire appel au lecteur pour qu'il fasse passer à l'existence objective le dévoilement que j'ai entrepris par le moyen du langage " ${ }^{87}$. À quoi l'écrivain en appelle-t-il ? À la liberté créatrice du lecteur. Nulle contrainte, fascination ou 
supplique, qui nous ramèneraient au monde de l'intersubjectivité aliénée (que nous allons analyser dans un instant). La liberté créatrice s'offre comme valeur à réaliser par la liberté du lecteur ${ }^{88}$.

On est donc très loin de l'interprétation de la création esthétique de L'Être et le Néant, où elle était pensée, comme nous l'avons vu, dans l'horizon du désir d'appropriation. «Le rapport de création, écrivait Sartre, [...] constitue originellement l'appropriation ${ }^{89}$. Ici en revanche le lecteur donne généreusement ce que l'écrivain lui demande sans exigence. L'exigence en effet nous précipite dans le monde de l'aliénation, et c'est ce que nous devons maintenant brièvement analyser.

2.2. Le monde de l'aliénation

Avec "l'aliénation", comme le précise Sartre dans son plan de morale ontologique, nous sommes bien sur le terrain de l'ontologie et non sur le terrain historique de "l'oppression $"{ }^{90}$. C'est avec ce dernier concept seulement que la morale ontologique laisse apercevoir des perspectives historiques. La suppression de l'aliénation, corrélativement, n'est pas la révolution comme l'auraient dit Marx ou Engels, mais bien la «conversion", concept qui nous laisse toujours dans l'horizon de l'ontologie - il faudrait même dire : de l'onto-théologie, puisqu'il s'agit pour l'homme, comme nous venons de le voir, de se reconquérir comme liberté « créatrice».

Les trois grands types d'aliénation de la liberté de l'individu, précise Sartre, sont: l'aliénation a) par la nature humaine, b) par les devoirs et droits, c) par valeurs posées par l'Autre. À quoi Sartre ajoute l'aliénation à l'Ego et à Dieu, dont il fait semble-t-il le prototype même de toutes les aliénations ${ }^{91}$. Nous allons nous intéresser à l'impact sur la théorie de l'aliénation de la nouvelle définition de l'homme comme liberté créatrice. De ce point de vue l'allusion à l'Ego, qui nous ramène directement à l'article rédigé à Berlin, est significative : en réinterprétant maintenant la « dégradation» que subit la spontanéité créatrice transcendantale lorsqu'elle s'objective en Ego sous l'effet de la réflexion impure ${ }^{92}$, comme "aliénation", elle suggère, symétriquement, que l'«aliénation" dans les Cahiers pour une morale doit être comprise essentiellement comme une sorte de " réification ${ }^{93}$ " où se perd la créativité de la conscience humaine. De ce point de vue, il est tout à fait logique de voir apparaître comme première figure de l'aliénation celle que Sartre appelle aliénation par la nature humaine. Concernant les deux autres formes d'aliénation - par les devoirs et les droits et par les valeurs posées par l'autre -, il faut pour les analyser de plus près nous reporter aux pages du «Premier Cahier » consacrées aux « types de demande à l'autre " ${ }^{94}$.

"La prière, dit Sartre, s'adresse à Dieu où à l'homme " ${ }^{95}$ - on pourrait dire, par rapport au problème qui nous occupe, qu'elle s'adresse à autrui comme à Dieu dont on solliciterait humblement la bonne volonté tout en la captant sournoisement. La captation aliénante se masque derrière l'humble requête de l'esclave soumis. Comme dans le cas du séducteur faisant miroiter à la femme mariée une liberté dont il la prive dans l'instant même où il la lui promet, parce que précisément il captive cette liberté par ses prières. De sorte que la libre invention d'une réponse à ses avances disparaît en se révélant pure illusion ${ }^{96}$. De manière analogue, en sollicitant d'autrui sa pitié par la présentation de ma déchéance fascinante, je lui vole sa liberté dans l'instant même où je la sollicite.

58 La très longue étude de l'" exigence " ${ }^{97}$ confirme ces remarques en donnant l'occasion à Sartre de s'attaquer durement au monde des devoirs et des droits en tant que monde par excellence où la liberté créatrice de l'individu se perd définitivement. Comme 
c'était prévisible, la morale kantienne de l'impératif catégorique fait l'objet d'une charge violente, consistant à réinterpréter le devoir inconditionné comme insupportable oppression. Le rapport du sujet moral à la loi est réinterprété comme celui de l'esclave au maitre ${ }^{98}$. L'« obligation » doit donc être soigneusement distinguée de la " valeur ». La seconde, explique Sartre " hante la liberté créatrice " 99 . La première s'oppose à cette même liberté, elle « ne se découvre pas nécessairement, dit Sartre, à ma spontanéité : elle peut naître en dehors d'elle ou contre elle. Je la rencontre au lieu de la soutenir ${ }^{100}$. De l'obligation, Sartre dit : «Elle se donne comme s'existant. [...]. Elle pour moi transcendance transcendante " ${ }^{101}$, voulant suggérer sans doute par là qu'elle surplombe la liberté humaine en lui imposant une normativité radicalement transcendante où toute inventivité s'efface définitivement. "L'obligation me transcende vers sa propre fin : elle me traverse. Elle a un rapport extatique avec une fin et je suis dans le circuit traversé comme un courant électrique traverse un filament de carbone [...]. La fin de l'obligation est un butoir réel et étranger contre lequel je viens donner ${ }^{102}$. De nouveau, c'est la situation du maître et de l'esclave qui guide l'analyse. "Quand je dis : j'exige, je crée par-derrière le libre projet de l'esclave ma propre liberté comme vouloir inconditionné qui le guide " ${ }^{103}$. La liberté de l'esclave, par le fait même se trouve, pour reprendre l'expression décisive de La Transcendance de l'ego, " dégradée ». La révolte en conséquence est pensée comme destruction de toute forme d'obligation ${ }^{104}$.

3. Perspectives historiques

59 Enfin il y a un dernier point à analyser : le rapport de la nouvelle morale à l'exigence de reconnaissance de chaque liberté par toutes les autres au sein d'une sorte de "cité des fins ", qui serait comme l'idée située à l'infini donnant son sens à toute l'histoire humaine ${ }^{105}$.

D'après le plan de la nouvelle morale de 1947-1948 fourni par Sartre lui-même, à la Section I exposant la morale ontologique aurait dû s'adjoindre une partie fournissant une théorie de l'histoire humaine. "L'histoire, écrit Sartre, implique la morale (sans conversion universelle pas de sens à l'évolution ou aux révolutions). La morale implique l'Histoire (pas de moralité possible sans action systématique sur la situation) » ${ }^{106}$. Quoique cette implication réciproque de l'histoire et de la morale soit ainsi fermement exigée, elle ne va pas sans poser d'importants problèmes - pour autant que l'on puisse reconstituer une véritable théorie de l'histoire humaine dans les Cahiers, à partir de fragments non coordonnés et très lacunaires.

Du point de vue qui ici nous occupe, la difficulté principale prend la forme suivante: peut-on élaborer une doctrine satisfaisante de l'historicité humaine à partir de l'interprétation - qui fonde toute la morale ontologique - de l'homme comme liberté créatrice aliénée, mais susceptible, par "conversion", de se reprendre sur cette aliénation? Cela n'est aucunement évident.

Comment l'histoire apparaît-elle dans les Cahiers de 1947-1948? Il est difficile de s'en faire une idée d'ensemble, tant les indications du texte sont fragmentaires. On peut prendre comme fil conducteur le fait que Sartre, dans de très nombreux passages, se confronte à la conception hégélienne, qu'il qualifie de « sommet de la philosophie » ${ }^{107}$, tout en lui reprochant, nous allons y venir, une erreur capitale. Une rapide discussion du rapport de Hegel à Sartre va nous permettre de comprendre le lien que ce dernier souhaite établir entre morale et histoire en 1947-1948. Notons que Sartre ne se donne pas les moyens d'une discussion précise de la théorie hégélienne et marxiste, faute 
d'une lecture suffisante des principaux textes concernés ${ }^{108}$. Il ignore les Leçons sur la philosophie de l'histoire et n'évoque que brièvement les Principes de la philosophie de droit ${ }^{109}$. Il utilise principalement la Phénoménologie de l'esprit - et encore souvent indirectement, c'est-à-dire par la lecture des commentaires d'A. Kojève et de J. Hyppolite. À Hegel Sartre fait constamment ce reproche d'avoir conçu l'histoire humaine comme une totalité virtuellement totalisée et non pas comme une totalité irréductiblement détotalisée. "La collectivité historique, écrit-il par exemple dans les notes dispersées qui ouvrent le "Premier Cahier ", est une totalité détotalisée " ${ }^{110}$. Impossible donc, comme le prétend Hegel, de ressaisir les événements apparemment multiples, les hasards, les discontinuités, dans la présence à soi d'un Esprit absolu toujours déjà là en puissance dès les commencements pour introduire, en deçà des apparences, la continuité, l'unité, la nécessité dialectique. Sartre conteste vigoureusement la tentative hégélienne de «retrouver, par delà la diversité, des consciences chacune autre que les autres, l'unité de l'objet, c'est-à-dire de l'Esprit. L'esprit comme substance est derrière les consciences et se réalise par elles, elles en sont les modes spinozistes. La conscience alors devient épiphénomène " ${ }^{11}$. Le hasard, affirme au contraire Sartre, est en histoire un irréductible ${ }^{112}$, tout de même que la multiplicité ${ }^{113}$ et la discontinuité ${ }^{114}$. La dialectique de l'histoire est donc une illusion, car elle suppose la croyance en une impossible totalité existant en puissance dans les événements dispersés.

Or ces considérations sur l'histoire humaine se relient étroitement au problème moral : la totalisation de l'humanité ne peut apparaitre, au sein de cette détotalisation permanente qu'est l'histoire réelle, que comme exigence, ou, si l'on préfère que comme cette idée, située à l'infini, d'un règne (ou d'une cité) des fins. En ce se sens l'histoire humaine ouvre sur l'infini ${ }^{115}$. Cette thématique, très présente dans les Cahiers, est apparemment issue assez directement de la philosophie kantienne de l'histoire. En même temps cependant Sartre cherche à distinguer sa propre conception de la cité des fins de celle de Kant. Par exemple dans le passage du "Premier Cahier " consacré à l'étude de la violence, Sartre montre comment Trotski accepte la position d'une fin absolue de l'histoire qui le met dans une proximité dangereuse à Kant et à sa morale bourgeoise de l'impératif. Cette fin, chez Trotski, c'est « l'accroissement du pouvoir de l'homme sur la nature et [...] l'abolition du pouvoir de l'homme sur l'homme » ${ }^{116}$. Sartre commente : «Je retrouve ici un idéal kantien : c'est la cité des fins ». Mais la difficulté justement c'est, tout en acceptant cette idée d'un « règne des fins » ou d'une « cité des fins »- expressions que Sartre reprend à son propre compte en plusieurs endroits des Carnets ${ }^{117}$-, de saisir que l'histoire n'a pas une direction vers un "impératif " susceptible d'être "réalisé intemporellement par le pur accord des bonnes volontés " ${ }^{118}$. La fin de l'histoire ne doit pas être comprise comme Idée "éternelle et transcendante à l'histoire", mais comme le sens mouvant de l'entreprise humaine, se réalisant - toujours partiellement - dans cette entreprise même.

En ce sens donc, et pour revenir à notre discussion entre Sartre et Hegel: si l'histoire était une totalité virtuellement totalisée, elle ne s'articulerait aucunement à une morale. Cela peut tout à fait se comprendre, mais l'interprétation sartrienne de l'histoire qui se dessine ici enveloppe une conception de l'action historique qui ne s'accorde pas très bien avec celle de l'action humaine comme "créatrice " dans la «morale ontologique». Dans le cadre de cette morale, l'histoire humaine peut être pensée, disons pour faire bref, comme jeu de l'aliénation et de la désaliénation de cette " créativité ». Mais de son côté la théorie de l'histoire issue de la discussion critique de 
Hegel se présente fort différemment, par sa conception en particulier de l'action historique et de l'« oppression ».

Concernant la conception de l'action historique, disons en simplifiant qu'elle n'implique plus d'appréhender l'action humaine comme "créativité absolue susceptible de "dégradation" ", mais plutôt comme "entreprise " engagée par ses projets dans les situations concrètes pour les modifier. Les actions historiques sont des " projets ouverts " ${ }^{119}$ engagés dans des rapports essentiellement mouvants avec le monde. Qu'il n'y ait pas ici trace de "créativité » n'implique nullement que l'on soit dans le monde de l'aliénation et de l'inauthenticité, au contraire. Sartre dit plutôt que c'est authenticité pour l'homme que de s'apparaitre, dans la réflexion non complice, comme "ensemble mouvant de perpétuelle mise en question et de perpétuel dépassement " ${ }^{120}$. L'action historique, écrit Sartre en ce sens, est " libre dépassement et invention avec les moyens $d u$ bord ${ }^{121}$. Il faut risquer, prendre sa chance, dans une certaine ignorance de tous les tenants et aboutissants la situation ${ }^{122}$. Si l'existant humain présente une certaine cohérence dans sa vie, ce n'est aucunement une cohérence d'être, mais une cohérence d'entreprise, que Sartre appelle «l'accord avec soi» ${ }^{123}$. En assumant son «être diasporique " ${ }^{124}$ et sa radicale finitude l'existant authentique s'ouvre à autrui et au monde ${ }^{125}$ en abandonnant tout désir de le capter dans les rets de son désir de création ${ }^{126}$. Il devient capable de "solidarité », comme lorsqu'il donne à boire à un misérable qui a soif, non pas pour flatter son propre Ego, dans un mouvement centripète d'amour-propre, mais en s'engageant pour l'autre par pure générosité ${ }^{127}$, dans une entreprise essentiellement risquée, en ce sens que l'autre va y répondre de manière totalement imprévisible.

D'autre part la redéfinition de l'historicité humaine, hors de toute thématique abstraite de l'aliénation de la créativité absolue, comme entreprise mouvante où le projet (pour reprendre la formule de Merleau-Ponty) s'engrène sur la situation, ouvre des perspectives sur une théorie de l'«oppression » où l'être humain ne peut voir ses projets lui être volés par la violence de son semblable que dans des situations concrètes marquées historiquement et socialement. L'enfant ne peut être opprimé par son père que dans une situation socio-historique particulière, celle par exemple de la famille bourgeoise du XIX ${ }^{e}$ siècle. Le prolétaire ne peut être opprimé par le capitaliste que dans la situation concrète d'un certain mode de production historique où la force de travail peut être librement mise sur le marché. L'oppression par le mensonge se réalise dans les circonstances particulières du rapport, par exemple entre les dirigeants d'un parti et les simples militants, ou entre des dirigeants religieux et le peuple ignorant.

Quelques mots pour conclure. Au terme de ces analyses, on s'aperçoit d'abord que toutes les variantes de morales liées à l'ontologie - que ce soit sous la forme d'une subordination de l'agir à l'être, comme dans les tentatives qui vont jusqu'à L'Être et le Néant, ou à l'inverse d'une subordination de l'être à l'agir comme dans les Cahiers de 1947-1948, ont fait lever sous les pas de Sartre d'innombrables apories. Concernant la " morale ontologique » de 1947-1948, son articulation à la théorie de l'histoire humaine que Sartre commence d'ébaucher reste largement problématique. Il est vrai que Sartre à l'époque, faute en partie d'une lecture sérieuse de Marx, ne possède pas encore de théorie nettement affirmée de l'histoire - cette théorie ne se formulera que dans la Critique de la raison dialectique, en 1960. Mais cette dernière œuvre ne résolut nullement la question épineuse de l'articulation de l'histoire et de la morale. En témoignent les recherches inabouties de 1964-1965, dont nous avons un écho grâce à la publication : 
Sur les écrits posthumes de Sartre ${ }^{128}$. Ce que nous entrapercevons de "l'éthique dialectique » sartrienne grâce à cette publication nous laisse deviner que l'articulation de la morale individuelle de la conversion radicale, et de l'analyse du conditionnement socio-historique de toute praxis, demeure largement problématique.

\section{NOTES}

1.. Décade de Cerisy-la-Salle, « Jean-Paul Sartre, écriture et engagement », 20-30 Juillet 2005 ; notre communication portait sur : «L'ontologie et les perspectives morales ». 2. Cahiers pour une morale, Paris, Gallimard, 1983. (Abréviation ultérieure : $C D G$ ). L'essentiel de cet important texte (inédit du vivant de Sartre) se compose de deux «Cahiers ». Le premier a pour centre une tentative de penser l'histoire humaine en se dégageant de l'emprise hégélienne. Le second est consacré pour essentiel à l'élucidation du concept de « conversion », clé de la « morale ontologique » de 1947-1948.

Pour ce qui est de la compréhension des «Cahiers », je dois rappeler ici ma dette à l'égard de Gérard Wormser, qui, dès leur publication, en 1983, a commencé une étude de longue haleine sur ce texte, dans le cadre du « Séminaire de phénoménologie » de l'E.N.S. de Saint-Cloud dirigé par B. Besnier, auquel je participai aussi, mais avec d'autres projets de recherche.

3.. Les Mots, Paris, Gallimard-Folio, 1981, p. 214.

4.. OR, p. 210.

5.. CDG, p. 495.

6.. CDG, p. 318.

7.. CDG, p. 244. Voir aussi p. 321.

8.. $C D G$, p. 313.

9.. $C D G$, p. $316,317$.

10.. CDG, p. 224-225. Désirer être son propre fondement, c'est-à-dire désirer la valeur, signifie exactement : désirer n'être ni libre ni situé.

11.. Une seule exception à cette règle, mais que Sartre avoue très explicitement, l'étude de la responsabilité dans la quatrième Partie de L'Être et le Néant ressortit bien à la morale et non à l'ontologie.

12.. EN, p. 111, n. 1. Il est donc à notre avis inexact d'assimiler comme le fait P. Cabestan (" Authenticité et mauvaise foi : que signifie être soi-même? ", Les Temps modernes, « Notre Sartre », Juil.-Oct. 2005, p. 604-626) les concepts de mauvaise foi et d'inauthenticité dans L'Être et le Néant. La mauvaise foi désigne un ensemble de conduites existentielles présentes nécessairement en tout être humain (donc non qualifiables moralement), en ce sens que leur fondement est la structure existentiale de la présence (à) soi. Il s'agit pour l'homme de jouer avec la liberté facticielle qu'il « est ». L'inauthenticité en revanche désigne certaines conduites existentielles choisies par tel ou tel être humain (donc qualifiables moralement, en l'occurrence négativement), et caractérisées par le désir de fuir la liberté facticielle que l'homme « est ». 
13.. L'« authenticité » est définie de manière énigmatique ( $E N, \mathrm{p} .111, \mathrm{n} .1)$ comme une « reprise de l'être pourri par lui-même » permettant à l'homme d'échapper à la mauvaise foi.

14.. EN, p. 722 (soulignement modifié).

15.. EN, p. 669.

16.. EN, p. 720.

17. L'expression de « responsabilité absolue » est à la page 639 de L'Être et le Néant. Dans sa forme classique, la responsabilité n'a de sens que relatif : l'individu n'est responsable que de telle ou telle action déterminée et il est responsable à tel ou tel degré. En droit pénal, c'est telle action déterminée délictueuse qui est imputée à l'individu, et dans certaines limites.

18.. EN, p. 638-643: «Liberté et responsabilité ».

19.. EN, p. 614, 632. Nous montrons dans un livre à paraître (La Première Philosophie de Sartre) que ce concept de « liberté absolue » est l'un des quatre attracteurs métaphysiques du champ de l'ontologie phénoménologique dans L'Être et le Néant. Ce concept reprend certains éléments du la notion de « spontanéité » absolue de $L a$ Transcendance de l'ego, " se recréant » à chaque instant " « ex nihilo » tout en créant le monde (dans une grande proximité, donc, au Dieu cartésien).

20.. MERLEAU-PONTY, Phénoménologie de la perception, Paris, Gallimard, 1945, p. 505.

21.. EN, p. 641.

22.. EN, p. 639-640.

23.. EN, p. 639.

24.. EN, p. 720.

25.. EN, p. 669.

26.. S. de BEAUVOIR, La Force de l'âge, Paris, Gallimard-Folio, t. 1, 1980, p. 23.

27.. EN, p. 77.

28.. Sartre emploie (p. 690) l'expression de « réflexion purifiante », qui équivaut chez lui au concept de conversion à la vie bonne par abandon de la vie mauvaise ou règne la " réflexion complice » (celle qui occulte la liberté absolue de l'individu). L'opposition entre les deux espèces de réflexion apparaît déjà dans La Transcendance de l'ego.

29.. EN, p. 722.

30.. CM, p. 484-487.

31.. Sartre définit cette aliénation : « l'aspect social de la réification » ( $C M$, p. 485), suggérant ainsi le lien essentiel entre morale et histoire.

32.. CM, p. 486.

33.. Les valeurs les plus basses « écrasent la liberté sous l’Être » (pureté, innocence, race, sincérité) ; les valeurs vitales sont des valeurs « intermédiaires » (noblesse, virilité, valeurs sexuelles, et de nouveau race) ; les « valeurs sociales » (nation, société, sacrifice) sont ambiguës : « l'autre comme produit du projet et comme sollicitation extérieure du projet »; les « valeur de subjectivité » (générosité, responsabilité, création, esprit critique, passion) sont les suprêmes valeurs en ceci qu'elles expriment l'exigence de recréation de soi inscrite dans la liberté humaine ; quant à cette liberté elle-même se situe au delà de ce plafond des valeurs de subjectivité.

34.. CM, p. 486.

35. Toutefois dans La transcendance de l'Ego (cf. p. 48) la réflexion " pure » se relie bel et bien à une thématique de reconquête par la spontanéité transcendantale de sa capacité de se recréer ex nihilo.

36.. EN, p. 249. 
37.. Dans L'Être et le Néant, la réflexion pure est essentiellement un concept ontologique, désignant une manière spécifique pour le pour soi de dévoiler l'être qui est le sien. Dans les Cahiers, elle n'est pas " pure contemplation mais elle-même projet » (CM, p. 490 ; voir aussi p. 495). Quelques références. L'Être et le Néant oppose : d'un côté la réflexion «pure » $(E N$, p. $201,207,722)$ ou « purifiante » $(E N$, p. 249$)$; de l'autre la réflexion « impure » (EN, p. 201, 206, 207), c'est-à-dire objectivante. Dans les Cahiers, on note les expressions suivantes : « réflexion complice » $(C M$, p. 12-13, 471, 489); réflexion « impure » (CM, p. 18), réflexion non complice » (CM, p. 486).

38.. CM, p. 427-483.

39.. CM, p. 484-487.

40.. CM, p. 488-570.

41.. CM, p. 487. C'est la conception hégélienne qui est principalement visée, Marx est souvent cité, mais sa conception de l'histoire et de l'aliénation ne fait pas l'objet de discussions approfondies. Le « Premier Cahier » consacre un assez long passage à la discussion de l'Anti-Dühring d'Engels (CM, p. 353 s.).

42.. Le terme apparaît en 1945, dans un article intitulé : «La libération de Paris : une semaine d'apocalypse ». Il y est défini ainsi (M. Contat, M. Rybalka, Les Écrits de Sartre, Paris, Gallimard, 1970, p. 125-126) : « une organisation spontanée des forces révolutionnaires... l'explosion de la liberté, la rupture de l'ordre établi et l'invention d'un ordre efficace et spontané ». Au début du « Second Cahier » (p. 429), Sartre évoque ce concept. Il désigne, dit-il, le moment de la fin de l'histoire par disparition de l'oppression. « Ainsi la liberté fait exploser perpétuellement l'idéologie, la mythologie et les rites antérieurs : elle réalise la libération par la conduite et l'idée neuve. C'est le moment de l'Apocalypse (c'est aussi le moment de la fête) ».

43.. Voir CM, p. 519. Observer autrui, explique Sartre, veut dire : « son acte est constitué avec une signification qu'il lui donne, sur le fond d'une signification que je lui donne à partir du monde». (Soulignement modifié).

44.. Voir par exemple CM, p. 528 : «L'En-soi-pour-soi est manqué ».

45.. «Sens de la conversion : rejet de l'aliénation» (CM, p. 486).

46.. « Sartre, Heidegger et la question de l'humanisme», in Sartre: du mythe à l'histoire, volume édité par G. Wormser aux éditions Sens Public/Editoo.Com (2005).

47.. L'artiste, comme on l'a vu, constitue un type humain dont tout le projet existentiel est de créer, mais Sartre précise bien (EN, p. 673), que cette entreprise vise un but contradictoire et par là même irréalisable.

48.. CM, p. 129. Dans le plan de morale ontologique, la création apparaît comme une conquête de la conversion ( $C M$, p. 486). «La modification du projet : la création. Donner un fondement à son être en le créant du dehors. But absolu : donner la liberté humaine comme fondement à l'être du monde. Mais ce but n'est pas donné, il est voulu ».

49.. En dépit de quelques passages comme celui-ci - aussi bien L'Être et le Néant est loin de constituer une œuvre totalement cohérente : « cet acte constamment renouvelé ne se distingue pas de mon être » (EN, p. 539).

50.. L'existentialisme est un humanisme, Paris, Nagel, 1970, p. 38 : «L'homme invente l'homme ».

51.. CM, p. 484.

52.. CM, p. 485. Sartre donne l'exemple de la situation - bien connue depuis L'Être et le Néant - du regard. « Je vois l'Autre qui me voit. J'affirme que j'existe comme l'Autre et comme je suis pour l'Autre. Mais l'Autre existe pour moi comme Autre et moi je suis 
pour lui l'Autre. C'est-à-dire l'objet transcendé. Je suis donc à partir de là pour moimême l'Autre ".

53.. Si l'on se fie à l'index établi par Jean-Gabriel ADLOFF (Sartre, Index du corpus philosophique, I, Paris, Klincksieck, 1981), le terme d'aliénation n'apparaitt que 10 fois dans L'Être et le Néant.

54.. Voir cette phrase qui clôt la critique de l'ouvrage d'ENGELS, L'Anti-Dühring, CM, p. 396 : « Loin que l'aliénation soit une des conséquences de l'oppression, elle en est un facteur ». On note dans le « Second Cahier » (CM, p. 524) une citation de Marx définissant l'« aliénation » de l'ouvrier par le fait que son travail devient en face de lui « une puissance autonome ». Sartre ne donne pas l'origine de cette citation, mais elle vient des « Manuscrits de 1844 » (traduction E. Bottigelli, Paris, Éditions sociales, 1972, p. 58).

55.. A. RENAUT (Sartre, le dernier philosophe, Paris, Grasset, 1993, p. 203 s.) affirme que le projet sartrien de morale s'effondre ici, victime de sa contradiction. Jugement excessif qui repose sur une interprétation contestable de l'individu, que Sartre comprendrait comme « monade » (op. cit., p. 225). Or dès La Transcendance de l'ego, Sartre affirme avec la plus grande netteté que la conscience créatrice - il s'agit dans ce texte de la conscience transcendantale - est individuée (voir TE, p. 23), mais qu'elle n'est aucunement une monade (voir TE, p. 26).

56.. Trois occurrences pour J.-G. ADLOFF (op. cit., p. 47), en fait quatre : p. 665, 672, 679, 680-681. Il y a bien une importante théorie de l'action libre dans L'Être et le Néant, mais jamais n'y apparaît cette formule surprenante des Cahiers (p. 503) : « l'action [...] est création ».

57.. Notre livre à paraître sur La Première Philosophie de Sartre donne toutes les explications souhaitables de ce concept d'attracteur métaphysique du champ ontophénoménologique.

58.. L'étude de la conversion constitue une bonne partie du « Second Cahier »; elle va de la page 488 à la page 569 du texte publié.

59.. CM, p. 542.

60.. CM, p. 492.

61.. EN, p. $664 \mathrm{~s}$.

62.. CM, p. 492-493.

63.. CM, p. 493-499.

64.. CM, p. 501.

65.. Questions II, Paris, Gallimard, 1948, p. 96. Sartre décrit ainsi l'activité du lecteur en tant qu'il collabore à la création de l'écrivain : « il pourrait toujours aller plus loin dans sa lecture, créer plus profondément ; et par là, l'œuvre lui paraît inépuisable et opaque comme les choses. Cette production absolue de qualités qui, au fur et à mesure qu'elles émanent de notre subjectivité, se figent sous nos yeux en objectivités imperméables, nous la rapprocherions volontiers de cette "intuition rationnelle" que Kant réservait à la Raison divine ».

66.. Cet inédit est maintenant disponible grâce à l'édition d'A. Elkaïm-Sartre chez Gallimard (Paris, 1989). Notons par exemple cette formule explicitant l'acte de perception : «je crée ce qui est » (op. cit., p. 49).

67.. Cette doctrine, affirme Sartre, est un mythe (CM, p. 499-500) - inverse du mythe de la causa sui.

68.. Vérité et existence, p. 19. 
69.. À la page 502 des Cahiers, Sartre écrit carrément : « Le Pour-soi est Dieu en ceci que s'il décide que l'Être a un sens, l'Être aura pour le Pour-soi ce sens ». Retour assez étrange aux anciennes convictions sur une possible divinisation de l'homme par la création, que Sartre avait pourtant fini par dépasser au cours de l'itinéraire difficile qui l'avait conduit de ses premiers écrits de jeunesse à L'Être et le Néant. Notons en passant que dans le texte intitulé La Liberté cartésienne, qui remonte à 1946, Sartre affirme que Descartes a commis l'erreur de projeter la liberté créatrice humaine en Dieu, de sorte que la vérité commande de rapatrier liberté créatrice divine en l'homme. (Cette thématique est feuerbachienne.)

70.. CM, p. 500.

71.. Ibid.

72.. CM, p. 502.

73.. Voir, par exemple, CDG, p. 403 : la lecture de Heidegger m'a enseigné

« l'authenticité et l'historicité ». " Historialisation » pourrait traduire le « Geschehen » heideggérien. «S'historialiser ", « historialiser », « historialisation », apparaissent dans L'Être et le Néant (p. 582, 604, 657).

74.. $C M$, p. 506. La formule qui vient un peu après - « Je ne puis exprimer mon époque qu'en la dépassant » - est proche de la problématique de la transcendance (ou de la liberté) située des Carnets et de la Conclusion de L'Imaginaire. Il en va de même de l'affirmation ( $C M$, p. 507) que l'authenticité consiste à reprendre sa gratuité à son compte. Voir aussi «Écrire pour son époque », fragment de « Qu'est-ce-que la littérature? ? publié en 1946.

75.. CDG, p. 507-509. Sauvons ce passage (p. 508) : « précisément parce que je suis gratuit, je peux m'assumer, c'est-à-dire non pas fonder cette gratuité qui restera ce qu'elle est, mais la reprendre à mon compte ».

76.. Sartre hésite entre des formules prudentes comme celle de la page 502 des Cahiers : « le pour soi a une tâche de quasi-création puisqu'il extirpe des ténèbres de l'indifférenciation ce qui par essence y retombe toujours ", et des affirmations énormes comme celle de la page 514 : « originellement l'homme est générosité, son surgissement est création du monde. Il n'est pas d'abord pour ensuite créer [...] mais dans son être même il est création du monde ». Voir aussi p. 510 : «L'homme se trouve héritier de la mission du Dieu mort : tirer l'être de son effondrement perpétuel dans l'absolue indistinction de la nuit ».

77.. CM, p. 513.

78.. À partir de la page 514 des Cahiers, Sartre entame l'étude du rapport aux autres hommes comme « créatures révélées ».

79.. CM, p. 515.

80.. CM, p. 515.

81.. Ibid. La thématique du regard pétrifiant réapparaît à plusieurs reprises, preuve que Sartre ne se résout pas totalement à l'abandonner. Voir par exemple aux pages 519-520 des Cahiers, la description d'un soldat qui voit son camarade s'avancer vers un piège tendu par l'ennemi, et dont il n'a nulle conscience ; pour l'observateur à l'abri, les mouvements du soldat en danger apparaissent dirigés par une intention totalement réifiée et ressaisie par l'Être.

82.. CM, p. 516.

83.. CM, p. 516.

84.. Situations II, Paris, Gallimard, 1968, p. 90.

85.. Ibid., p. 93. 
86.. Ibid., p. 93-94.

87.. Ibid., p. 96.

88. L'élucidation de la création esthétique subit ici l'attraction évidente de la morale.

« L'œuvre d'art n'a pas de fin, nous en sommes d'accord avec Kant. Mais c'est qu'elle est une fin » (Situations II, op. cit., p. 98).

89.. EN, p. 679. Voir aussi p. 681 : « Le rapport de création continuée enveloppe comme sa contradiction implicite l'indépendance absolue et en soi des objets créés. La possession est un rapport magique; je suis ces objets que je possède, mais dehors, face à moi ; je les crée comme indépendants de moi ; ce que je possède, c'est moi hors de moi, hors de toute subjectivité, comme un soi qui m'échappe à chaque instant et dont je perpétue à chaque instant la création ».

90.. CM, p. 485. Sur les types d'oppressions, voir par exemple : l'enfant dominé par ses parents, CM, p. 197-203 ; l'ignorance, CM, p. 306-314 ; l'ouvrier prisonnier de la religion bourgeoise, CM, p. 209-211.

91.. CM, p. 485. Sartre écrit que, au-delà de l'étude des trois modalités citées de l'aliénation, il faudrait s'attaquer à « la catégorie de toutes les Aliénations ». A. Renaut suggère d'interpréter cette expression (op. cit., p. 216) comme signifiant que Sartre vise ici une interrogation fondamentale sur la «catégorie même de l'Autre » - nous dirions plutôt : sur la catégorie de l'Autre-en-moi. D'après les allusions cursives de Sartre, l'Autre-en-moi, ce peut être, d'abord l'Ego, et ensuite, pour le croyant, Dieu.

92.. Voir TE, p. 63-34: « une spontanéité bâtarde et dégradée ».

93.. CM, p. 484.

94.. CM, p. 224 s.

95.. CM, p. 225.

96.. $C M$, p. 240-243.

97.. CM, p. 248-285.

98. CM, p. 250.

99.. CM, p. 258. (En vérité il n'est pas du tout sûr que la thématique de la valeur hantant le circuit de l'ipséité facticielle et finie se transpose si aisément à la doctrine de la liberté humaine créatrice).

100.. CM, p. 261-262.

101.. CM, p. 262.

102.. Ibid.

103.. CM, p. 273.

104.. CM, p. 418 : « Puisque l'esclavage est l'ordre, la liberté sera désordre, anarchie, terrorisme $»$.

105.. « Cité des fins », voir $C M$, p. 169, 171, 177. « Règne des fins », voir $C M$, p. 487.

106.. CM, p. 487.

107.. CM, p. 67.

108. Bien que Marx et Engels ne soient nullement ignorés, ils ne sont jamais au centre de la construction d'une doctrine de l'histoire dans les Cahiers - et ce, même sur le point où on aurait pu attendre une confrontation : la question de l'oppression. Sartre écrit bien ( $C M$, p. 485) : « Critique du marxisme : l'aliénation précède l'oppression », mais en pratique, il développe son analyse de l'oppression sans véritablement la confronter à celle de Marx ou d'Engels. À la page 67 des Cahiers, Sartre affirme que, par rapport à ce sommet de la philosophie qu'est Hegel, le marxisme n'est qu'une dégénérescence. L'article « Matérialisme et révolution » (1946) ne s'en prenait que de manière assez globale à l'idée d'une dialectique purement matérielle.

109.. CM, p. 148. 
110.. CM, p. 26 .

111.. $C M$, p. $446-447$.

112.. CM, p. 30, 33, 37 (« la face de hasard de tout événement historique ») ; p. 40 (« ce qui est subjectivité orientée, vu du dedans du groupe, est hasard pour l'autre groupe »); p. 44 (« c'est la statistique [...] qui décide de l'orientation dialectique qui fait l'histoire »); p. 65 (« toute liberté est transcendée par les autres libertés, elle devient hasard pour les autres, et son action devient objet. De fait il y a un caractère statistique de l'histoire »).

113.. CM, p. 92 : «L'histoire habitée par le mythe de l'unification ». Il n'y pas réellement d'« évolution de la pluralité vers l'Unité » (ibid.). « L'Histoire, dit aussi Sartre (CM, p. 90) : structures extérieures les unes aux autres ». Voir aussi les concept de «structure fibreuse » de l'histoire et de « prolifération décentrée » (CM, p. 40, 76-77). 114.. « Histoire : continuité idéale perpétuellement brisée par le discontinu réel » $(C M$, p. 33).

115.. CM, p. 91.

116.. Leur morale et la nôtre, cité par les Cahiers à la page 169.

117.. En particulier dans l'esquisse du plan de la Section II de la morale (CM, p. 487) : « Rôle de l'homme dans l'histoire. La vraie moralité (concrète) : préparer le règne des fins par une politique révolutionnaire, finie et créatrice. Retournement : que le règne des fins est précisément dans la préparation du règne des fins ». Citons aussi cette phrase : « La fin de l'histoire ce serait l'avènement de la morale » (CM, p. 95). Il y a sur ce point une ambiguité dans les Cahiers, qui tendent à mettre l'accent soit sur l'aspect moral de l'idée située à l'infini d'une fin de l'histoire - il s'agit alors de l'idée de l'universelle reconnaissance de toutes les libertés dans le " règne des fins "; soit plutôt sur l'aspect politique de cette idée - par exemple (CM p. 109 ) lorsque Sartre affirme que la fin de l'histoire c'est « le socialisme ».

118. CM, p. 170 et 171.

119.. CM, p. 494.

120.. CM, p. 495. Et p. 110 : «Ainsi s'entrevoit, par delà l'antinomie de la morale et de l'Histoire, une morale concrète qui est comme une logique de l'action ».

121.. CM, p. 85.

122.. CM, p. 96.

123.. CM, p. 495.

124.. Ibid.

125.. Cette ouverture s'atteste dans des attitudes comme la générosité, l'amour, la sollicitude pour la fragilité d'autrui. Toutes attitudes que la doctrine de l'action créatrice certes peut penser, mais avec de grandes difficultés, comme on le voit dans le fait qu'il faut au fond tout le dispositif complexe et singulier liant l'artiste à ses lecteurs, spectateurs et auditeurs, pour faire apparaître au sein même de libertés créatrices quelque chose comme le don désintéressé et la réponse généreuse.

126.. Dans la réflexion purifiante le projet ne se récupère pas « contemplativement », mais « pratiquement». (CM, p. 495). La réflexion fait sien le projet « non par identification ou appropriation mais par assentiment et alliance. Autrement dit, la conversion consiste à renoncer à la catégorie d'appropriation, qui ne peut régir que les rapports du Pour-soi avec les choses, pour introduire dans le rapport interne de la Personne le rapport de solidarité, qui sera plus tard modifié en solidarité avec les autres » (ibid.). 
127.. Dans les Cahiers, la générosité apparaît à plusieurs reprise comme valeur suprême de la nouvelle morale.

128.. Éditions de l'Université de Bruxelles, 1987. « Éthique dialectique » est l'expression choisie par B. Stone et E. Bowman pour désigner les notes sartrienne inédites de 1964-65 revenant à l'éthique après l'élaboration de la philosophie dialectique de l'histoire. Sur les écrits posthumes de Sartre donne un résumé de la Conférence de Rome de 1964 et des Conférences préparées par Sartre pour l'Université de Cornell en 1965.

\section{RÉSUMÉS}

Dans la philosophie de Sartre, depuis les années d'études jusqu'à la rédaction des Cahiers pour une morale, ontologie et morale marchent pour ainsi dire du même pas. Parfois, les préoccupations ontologiques commandent le questionnement moral. C'est le cas lorsque la toute première métaphysique définit une morale du salut par l'art, ou encore lorsque la «métaphysique de la réalité-humaine » (1939-1940) engage à une réflexion sur l'authenticité ; ou bien enfin, dans L'Être et le Néant, quand les "perspectives morales" sont dégagées à partir des conclusions de l'ontologie phénoménologique. Parfois, à l'inverse, la construction d'une morale conduit à une refonte des concepts de l'ontologie. C'est le cas lorsque les Cahiers (1947-1948) redéfinissent l'être humain comme «créateur ». Cette seconde perspective, née des apories rencontrées par Sartre dans l'élaboration du premier chemin, connaît toutefois des difficultés. Il conduit à envisager toujours dans les Cahiers - d'aller vers la morale par l'histoire humaine comme "totalité détotalisée ». Cependant cette dernière tentative souffre d'une insuffisante élaboration du concept d'histoire. Cette lacune ne sera comblée qu'avec la Critique de la raison dialectique (1960). Mais ce sera au prix de l'ajournement de la question morale. Celle-ci ressurgit in fine vers 1964-1965 sous la forme très problématique d'une éthique dialectique.

In the philosophy of Sartre (from early on to the Cahiers pour une morale) ontology and morality go hand in hand. Sometimes, ontological research leads to a moral questioning; this is the case when his early metaphysics suggests a morality of salvation by art, or when the metaphysics of human reality (1939-1940) leads to a reflection about authenticity, or in L'Être et le Néant at last, when the "Perspectives morales" are drawn from the conclusion of the phenomenological ontology. Sometimes the establishment of a morality leads to a new ontological conceptualisation; this is the case when the Cahiers pour une morale (1947-1948) define the human being as "creator". This new approach leads to new problems, which suggest a new path to morality, human history as detotalized totality. However, this final approach lacks a good theory of history, which will be formulated in the Critique de la raison dialectique (1960). The moral questioning will nevertheless come back around 1964-1965 in the problematic form of dialectical ethics. 


\section{AUTEUR}

\section{ALAIN FLAJOLIET}

Alain Flajoliet est né en 1954. Ancien élève de l'E.N.S. de Saint-Cloud, agrégé et docteur en philosophie. Enseigne la philosophie en classes préparatoires. A participé à de nombreux ouvrages consacrés à Sartre : Sartre phénoménologue (Alter, $\left.\mathrm{n}^{\circ} 10\right)$, Sartre, désir et liberté (PUF), Dictionnaire Sartre (H. Champion), J.-P. Sartre, violence et éthique (Sens Public), Sartre et la phénoménologie (Fontenay/Saint-Cloud, E.N.S. Éditions), Retour critique sur L'Être et le Néant (Études sartriennes, $n^{\circ}$ 9). 